\title{
Self-modulation of nonlinear waves in a weakly magnetized relativistic electron-positron plasma with temperature
}

\author{
Felipe A. Asenjo" \\ Institute for Fusion Studies, University of Texas at Austin, Austin, Texas 78712, USA \\ Felix A. Borotto \\ Departamento de Física, Facultad de Ciencias Físicas y Matemáticas, Universidad de Concepción, Chile
}

\begin{abstract}
Abraham C.-L. Chian
Observatoire de Paris, LESIA, CNRS, 92190 Meudon, France, and National Institute for Space Research (INPE) and World Institute for Space Environment Research (WISER), P.O. Box 515, 12227-010 São José dos Campos-SP, Brazil

Víctor Muñoz and J. Alejandro Valdivia

Departamento de Física, Facultad de Ciencias, Universidad de Chile, Casilla 653, Santiago, Chile, and Centro para el Desarrollo de la Nanociencia y la Nanotecnología, CEDENNA

Erico L. Rempel

Institute of Aeronautical Technology (ITA), 12228-900 São José dos Campos-SP, Brazil and National Institute for Space Research (INPE) and World Institute for Space Environment Research (WISER), P.O. Box 515, 12227-010 São José dos Campos-SP, Brazil
\end{abstract}

(Received 28 November 2011; published 5 April 2012)

\begin{abstract}
We develop a nonlinear theory for self-modulation of a circularly polarized electromagnetic wave in a relativistic hot weakly magnetized electron-positron plasma. The case of parallel propagation along an ambient magnetic field is considered. A nonlinear Schrödinger equation is derived for the complex wave amplitude of a selfmodulated wave packet. We show that the maximum growth rate of the modulational instability decreases as the temperature of the pair plasma increases. Depending on the initial conditions, the unstable wave envelope can evolve nonlinearly to either periodic wave trains or solitary waves. This theory has application to high-energy astrophysics and high-power laser physics.
\end{abstract}

DOI: 10.1103/PhysRevE.85.046406

PACS number(s): 52.27.Ep, 52.27.Ny, 52.35.Mw, 52.35.Sb

\section{INTRODUCTION}

Relativistic electron-positron plasmas are of great interest in the study of high-energy astrophysical phenomena and future high-intensity laser experiments in laboratory. In astrophysics, it has been suggested that electron-positron plasmas can be found in pulsar magnetospheres [1], magnetars [2], accretion disks [3], the early universe [4], and supernovae [5], just to cite some examples. Observational evidence of astrophysical electron-positron plasmas has been obtained in the Cygnus-X1 x-ray binary system, the jet in the M-87 giant elliptical galaxy, and other x-ray sources in the Galactic Center [6-8]. Laboratory experiments capable of producing high-temperature electron-positron plasmas have not been performed so far. However, such experiments may be achievable using high-power ultrashort laser pulses in the future [9-11].

Relativistic nonlinear circularly polarized electromagnetic waves in a cold plasma were treated by Lünow [12], Clemmow [13], Kennel and Pellat [14], Chian and Miranda [15], and Chian [16] by seeking the exact traveling-wave solution of the governing equations. This problem was applied to a relativistic

\footnotetext{
*faz@physics.utexas.edu; this work was partially done at the Departamento de Ciencias, Facultad de Artes Liberales, Universidad Adolfo Ibáñez, Diagonal Las Torres 2640, Peñalolén, Santiago, Chile.
}

hot magnetized electron-positron plasma by Chian [17] and Asenjo et al. [18] using two different formalisms, but the same nonlinear dispersion relation was obtained.

The nonlinear phenomena in unmagnetized relativistic plasmas, such as solitons, have been studied previously in several times for different plasma species and for different field configurations using fluid models (for example, see Refs. [19-24] and references therein). The contribution of this paper is to study the self-modulation of a nonlinear circularly polarized electromagnetic wave in an electron-positron plasma with fully relativistic temperatures in a weakly magnetized regime. The self-modulation of a relativistic nonlinear circularly (and linearly) polarized electromagnetic wave in an electron-positron plasma was first investigated by Chian and Kennel [1] in the cold unmagnetized case. A large number of papers have improved the original model by Chian and Kennel [1]. For example, the existence of Alfvén vortices in the presence of a strong magnetic field was demonstrated by $\mathrm{Yu}$ et al. [25]; the influence of a strong magnetic field and ions was analyzed by Rizzato et al. [26]; and the effects of relativistic temperatures and phonon damping were considered by Gratton et al. [27].

The paper is structured as follows. First, in Sec. II, the fluid model for a relativistic plasma with a finite temperature is presented. Then, in Sec. III, the dispersion relation of a circularly polarized electromagnetic wave propagating along the background magnetic field in a relativistic electron-positron 
plasma is discussed. Next, in Sec. IV, a nonlinear Schrödinger equation for the complex wave amplitude is derived in the weakly magnetized and small-amplitude regime. In Sec. V, the modulational instability of the wave is studied. Finally, in Sec. VI, the results are summarized.

\section{RELATIVISTIC PLASMAS WITH A FINITE TEMPERATURE}

It is possible to formulate a theory for hot relativistic plasmas in a covariant form [28] where the fluid and the electromagnetic fields are unified into a single field. Here we will consider only the spatial part of those equations. Within this context the plasma dynamics is given by Maxwell's equations, the continuity equation

$$
\frac{\partial}{\partial t}\left(n_{j} \gamma_{j}\right)+\vec{\nabla} \cdot\left(n_{j} \gamma_{j} \vec{v}_{j}\right)=0
$$

and the evolution equation for each fluid $[18,28]$

$$
\left(\frac{\partial}{\partial t}+\vec{v}_{j} \cdot \vec{\nabla}\right)\left(f_{j} \gamma_{j} \vec{v}_{j}\right)=\frac{q_{j}}{m}\left(\vec{E}+\frac{\vec{v}_{j}}{c} \times \vec{B}\right)-\frac{1}{n_{j} m \gamma_{j}} \vec{\nabla} p_{j},
$$

where $\vec{v}_{j}, \gamma_{j}, q_{j}, p_{j}$, and $n_{j}$ are the velocity, relativistic Lorentz factor, charge, pressure, and rest-frame number density of each fluid, respectively; $m$ is the electron mass, $c$ is the speed of light, and $\vec{E}$ and $\vec{B}$ are the electric and magnetic fields. The $f_{j} \equiv f\left(T_{j}\right)$ function is related to the temperature $T_{j}$ in the form $f\left(T_{j}\right)=K_{3}\left(m c^{2} / k_{B} T_{j}\right) / K_{2}\left(m c^{2} / k_{B} T_{j}\right)$, where $K_{3}(x)$ and $K_{2}(x)$ are modified Bessel functions of order 3 and 2 , respectively [28], and $k_{B}$ is the Boltzmann constant. We use the subindex $j=e$ for the electron fluid and $j=p$ for the positron fluid.

In order to close the system of Eqs. (1) and (2) we consider the equation of state $p_{j}=n_{j} k_{B} T_{j}$ for an ideal gas.

We will write $q_{j}=-\eta_{j} e$, where $e$ is the positron charge, and $\eta_{e}=1$ and $\eta_{p}=-1$. Also, the electromagnetic fields can be written in terms of the scalar potential $\phi$ and the vector potential $\vec{A}$, as $\vec{E}=-\vec{\nabla} \phi-\partial_{t} \vec{A} / c$ and $\vec{B}=\vec{\nabla} \times \vec{A}$. The evolution of the electromagnetic four-vector potential $A^{\mu} \rightarrow$ $(\phi, \vec{A})$ can be obtained from the covariant wave equation $\partial_{\nu} \partial^{v} A^{\mu}=4 \pi / c J^{\mu}$, where $J^{\mu}=e n_{p} U_{p}^{\mu}-e n_{e} U_{e}^{\mu}$ is the four current, and $U_{j}^{\mu} \rightarrow\left(c \gamma_{j}, \gamma_{j} \vec{v}_{j}\right)$ is the four-velocity of each species. Hence, the spatial part of the covariant wave equation for the potential $A^{\mu}$ can be written as

$$
\frac{\partial^{2} \vec{A}}{\partial t^{2}}-c^{2} \nabla^{2} \vec{A}=4 \pi e c\left(n_{p} \gamma_{p} \vec{v}_{p}-n_{e} \gamma_{e} \vec{v}_{e}\right) \text {. }
$$

\section{CIRCULARLY POLARIZED ELECTROMAGNETIC WAVE}

It is well known that a circularly polarized electromagnetic wave, propagating along an ambient uniform magnetic field, is an exact solution of the classical and relativistic cold plasma fluid equations [12-16]. For relativistic hot plasmas, it has been shown $[17,18]$ that this is also an exact solution for constant, relativistic temperatures. In this case the thermal effects are introduced via the enthalpy-like quantity $f\left(T_{j}\right)$.

Indeed, following Ref. [18] we assume that all variables have a $(k z-\omega t)$ time and space dependence, so that a trans- verse circularly polarized electromagnetic wave of arbitrary amplitude

$$
\vec{A}=a_{0}[\cos (k z-\omega t) \hat{x}+\sin (k z-\omega t) \hat{y}]
$$

can propagate in this relativistic electron-positron plasma along the ambient uniform magnetic field $B_{0} \hat{z}$. Here $\omega$ is the frequency and $k$ is the wave number. For an electron-positron plasma, this purely transverse wave is an exact solution of the plasma equations of Sec. II. It has purely transverse circularly polarized fluid velocities associated with it (with $v_{z, j}=0$ ), so that for each fluid we have [18]

$$
\vec{v}_{j}=\eta_{j} \frac{e \vec{A}}{m c}\left(\frac{\omega}{f_{j} \gamma_{j} \omega+\eta_{j} \Omega_{c}}\right),
$$

where $\Omega_{c}=e B_{0} /(m c)$ is the cyclotron frequency.

For this transverse mode, the continuity equation (with $\vec{\nabla} \cdot \vec{v}_{j}=0$ ) requires that the laboratory frame densities $\gamma_{j} n_{j}$ be constant. Since the amplitude of the circularly polarized velocity is proportional to the constant amplitude of the circularly polarized vector potential field, the relativistic Lorentz factor

$$
\gamma_{j}=\left(1-\frac{\left|\vec{v}_{j}\right|^{2}}{c^{2}}\right)^{-1 / 2}
$$

is constant as well [18]. Therefore, the rest-frame densities $n_{j}$ will be constant.

We take the quasineutral approximation where we have that the densities in the laboratory frame are equal, $\gamma_{e} n_{e}=\gamma_{p} n_{p}$ [24]. Thus, $\vec{\nabla} \cdot \vec{E}=0$ and there is no longitudinal electric field accordingly with (4). This is consistent with $v_{z, j}=0$ and with $\vec{\nabla} p_{j}=0$, such as that $T_{j}$ and $f_{j}$ are constant by the equation of state.

Introduction of Eq. (5) in Maxwell's equations yields the dispersion relation for a circularly polarized wave $[17,18]$

$$
\frac{\omega^{2}-c^{2} k^{2}}{\omega}=\frac{\gamma_{e} \omega_{p e}^{2}}{f_{e} \gamma_{e} \omega+\Omega_{c}}+\frac{\gamma_{p} \omega_{p p}^{2}}{f_{p} \gamma_{p} \omega-\Omega_{c}},
$$

where $\omega_{p e}=\left(4 \pi e^{2} n_{e} / m\right)^{1 / 2}$ and $\omega_{p p}=\left(4 \pi e^{2} n_{p} / m\right)^{1 / 2}$ are the electron and positron plasma frequencies in their rest frames, respectively.

\section{NONLINEAR SCHRÖDINGER EQUATION FOR WAVE AMPLITUDE}

This fully nonlinear solution is obtained by solving the system of Eqs. (5)-(7). Notice that the solution depends on the amplitude of the electromagnetic wave through the vector potential.

In this paper we seek a solution in the weakly magnetized relativistic plasma limit $\left(\omega \gg \Omega_{c}\right)$. It turns out that we are able to find a nonlinear Schrödinger equation for the wave amplitude, with thermal effects introduced through the $f_{j}$ factor, thus improving on the previous results [1].

Noting that $f_{j} \geqslant 1$ always, the weakly magnetized limit is obtained when $f_{j} \omega \gg \Omega_{c}$. We use this limit in the denominator of the expressions for the fluid velocities [Eq. (5)], and then replace them in each relativistic Lorentz factor [Eq. (6)]. Hence, at the order $\Omega_{c}^{2} / \omega^{2}$, we obtain the equation for the 
relativistic Lorentz factors

$$
\gamma_{j}^{4}-\left(1+\frac{\lambda A^{2}}{f_{j}^{2}}\right) \gamma_{j}^{2}+\eta_{j} \frac{2 \Omega_{c} \lambda A^{2}}{\omega f_{j}^{3}} \gamma_{j}-\frac{3 \Omega_{c}^{2} \lambda A^{2}}{f_{j}^{4} \omega^{2}}=0,
$$

where $\lambda=e^{2} /\left(m^{2} c^{4}\right)$ and $A^{2}=\vec{A} \cdot \vec{A}$. In this limit it is possible to find approximate solutions for the Lorentz factors of each fluid, at the order $\Omega_{c}^{2} / \omega^{2}$ :

$$
f_{j} \gamma_{j}=\left(f_{j}^{2}+\lambda A^{2}\right)^{1 / 2}-\eta_{j} \frac{\Omega_{c} \lambda A^{2}}{\omega\left(f_{j}^{2}+\lambda A^{2}\right)}+\frac{3 \lambda A^{2} f^{2} \Omega_{c}^{2}}{2 \omega^{2}\left(f_{j}^{2}+\lambda A^{2}\right)^{5 / 2}} .
$$

The same results can be obtained by expanding $\gamma_{j}$ in the form $\gamma_{j}=g_{j 0}+g_{j 1}\left(\Omega_{c} / \omega\right)+g_{j 2}\left(\Omega_{c} / \omega\right)^{2}$, and using this in Eqs. (5) and (6) to find the coefficients $g_{j n}$. From (9), we notice that the Lorentz factors are different for the two species. This is because we are considering the weakly magnetized plasma limit where the symmetry between electrons and positrons with respect to the background magnetic field is broken. Thereby, because of that the quasineutral approximation holds, and the rest-frame densities of electrons and positrons are not equal.

We now need a closed expression for the velocities to insert in the right-hand side of Eq. (3). Using the relativistic factors of Eq. (9) in Eq. (5), the velocities are given, up to order $\Omega_{c}^{2} / \omega^{2}$, by

$$
\gamma_{j} \vec{v}_{j}=\eta_{j} \frac{e \vec{A}}{m c f_{j}}\left[1-\eta_{j} \frac{\Omega_{c}}{\omega\left(f_{j}^{2}+\lambda A^{2}\right)^{1 / 2}}+\frac{\Omega_{c}^{2} f_{j}^{2}}{\omega^{2}\left(f_{j}^{2}+\lambda A^{2}\right)^{2}}\right] .
$$

We treat the plasma as being in thermal equilibrium, $f_{e}=f_{p}=f$, so that we can write Eq. (3) as

$$
\frac{\partial^{2} \vec{A}}{\partial t^{2}}-c^{2} \frac{\partial^{2} \vec{A}}{\partial z^{2}}+\frac{2 \omega_{p}^{2} \vec{A}}{f}\left[1+\frac{\Omega_{c}}{\omega\left(f^{2}+\lambda A^{2}\right)^{1 / 2}} \Theta+\frac{\Omega_{c}^{2} f^{2}}{\omega^{2}\left(f^{2}+\lambda A^{2}\right)^{2}}\right]=0,
$$

where $2 \omega_{p}^{2} \equiv \omega_{p p}^{2}+\omega_{p e}^{2}$. This equation represents the nonlinear propagation of the circularly polarized electromagnetic wave packet. The factor $\Theta=\left(\omega_{p p}^{2}-\omega_{p e}^{2}\right) /\left(2 \omega_{p}^{2}\right)$ is a measure of the difference of the plasma frequencies in their respective rest frames. It is not null because the rest-frame densities are not equal. It can be calculated at first order in $\Omega_{c} / \omega$ using the neutrality condition $\gamma_{e} n_{e}=\gamma_{p} n_{p}$ and Eq. (9). With this condition it is straightforward to obtain $\Theta=-\left(\lambda A^{2} \Omega_{c} / \omega\right) /\left(f^{2}+\lambda A^{2}\right)^{3 / 2}$.

The nonlinear effects introduced by the relativistic Lorentz factors are present in the denominator $f^{2}+\lambda A^{2}$, which is a relativistic invariant. The function $f^{2}$ is a scalar because we define the temperature in the rest frame of the fluid. To define a circularly polarized electromagnetic wave we need a vector potential such that $\vec{\nabla} \cdot \vec{A}=0$, which in the Lorentz gauge implies that $\partial_{t} \phi=0$. This allows us to choose $\phi=0$, so that $A^{2}$ is also an invariant. Thus, all the terms in the square brackets in (11) are relativistic invariants.

In order to study the modulational instability, we now introduce a space-dependent complex modulational representation for the circularly polarized electromagnetic wave given by [1]

$$
\vec{A}=\frac{1}{2}\left[a(z, t) e^{-i \omega t}+\text { c.c. }\right] .
$$

Then from Eq. (11) it is possible to find an equation for $a(z, t)$. Taking the limit of a slowly time-varying modulation $\partial_{t}^{2} a \ll$ $\omega^{2} a$, we have

$$
i \frac{\partial a}{\partial t}+\frac{c^{2}}{2 \omega} \frac{\partial^{2} a}{\partial z^{2}}+\left\{\frac{\omega}{2}-\frac{\omega_{p}^{2}}{\omega f}\left[1+\frac{\Omega_{c}^{2}\left(f^{2}-\lambda|a|^{2}\right)}{\omega^{2}\left(f^{2}+\lambda|a|^{2}\right)^{2}}\right]\right\} a=0 .
$$

Equation (13) is for arbitrary wave amplitudes, and it admits stationary solutions. However, following Ref. [1], we treat the small-amplitude limit as an illustration. In this limit $f^{2} \gg$ $\lambda|a|^{2}$, and Eq. (13) yields

$i \frac{\partial a}{\partial t}+P(\omega) \frac{\partial^{2} a}{\partial z^{2}}+R\left(\omega,\left|a_{0}\right|^{2}\right) a+Q(\omega)\left(|a|^{2}-\left|a_{0}\right|^{2}\right) a=0$,

where

$$
\begin{gathered}
P(\omega)=\frac{c^{2}}{2 \omega}, \\
R\left(\omega,\left|a_{0}\right|^{2}\right)=\frac{\omega}{2}-\frac{\omega_{p}^{2}}{\omega f}-\frac{\omega_{p}^{2} \Omega_{c}^{2}}{\omega^{3} f^{3}}+Q(\omega)\left|a_{0}\right|^{2},
\end{gathered}
$$

and

$$
Q(\omega)=\frac{3 \lambda \omega_{p}^{2} \Omega_{c}^{2}}{\omega^{3} f^{5}}
$$

Note that we have added and subtracted a term proportional to the amplitude of the uniform circularly polarized wave $\left|a_{0}\right|^{2}$, to make sure that when $a(z, t)=a_{0} e^{i k z}$ we obtain the nonlinear dispersion relation of the uniform circularly polarized wave described in Sec. III, in the limits $a_{0}$ is constant, $\omega \gg \Omega_{c}$, and $f^{2} \gg \lambda\left|a_{0}\right|^{2}$. This guarantees that we have the proper boundary condition of finite amplitude in the limit $z-V t \rightarrow$ $\pm \infty$, where $V$ is the effective velocity of the wave packet. It is interesting to note that the same result can be obtained by expanding the wave equation in powers of $\partial / \partial_{t}, \partial / \partial_{z}$ and $\left(|a|^{2}-\left|a_{0}\right|^{2}\right)[29]$. 

as

The dispersion relation (7) can be solved for the frequency

$$
2 x^{2}=y^{2}+\frac{2}{f}+\left[\left(y^{2}+\frac{2}{f}\right)^{2}+\frac{8 \alpha}{f^{5}}\left(f^{2}-3\left|a_{0}\right|^{2} \lambda\right)\right]^{1 / 2},
$$

representing the electromagnetic branch [18], where $\alpha=$ $\Omega_{c}^{2} / \omega_{p}^{2}, x=\omega / \omega_{p}$, and $y=c k / \omega_{p}$. We note that this solution corresponds to a weakly magnetized electromagnetic wave that becomes the light wave $(\omega / k=c)$ in the limit $k \rightarrow \infty$ as shown in Ref. [18]. The nonlinear propagation of a circularly polarized electromagnetic wave packet for the Alfvén branch solution will be discussed elsewhere.

Equation (14) can be explicitly written as a nonlinear Schrödinger equation by changing $a \rightarrow a \exp \left(i R\left(\omega,\left|a_{0}\right|^{2}\right) t\right)$ :

$$
i \frac{\partial a}{\partial t}+P(\omega) \frac{\partial^{2} a}{\partial z^{2}}+Q(\omega)\left(|a|^{2}-\left|a_{0}\right|^{2}\right) a=0 .
$$

It is possible to construct solutions with other boundary conditions, for example, by letting $\left|a_{0}\right| \rightarrow 0$. We will see below another way that this can be accomplished.

In the above equation, the corrections for a relativistically thermal and weakly magnetized pair plasma are in the $Q(\omega)$ term and in $\omega$ through the dispersion relation Eq. (7). The $P(\omega)$ term has the same form as in the cold case. The temperature dependence on $Q$ is through the function $f$ in the denominator. In the low-temperature limit $k_{B} T \ll m c^{2}$, the function can be approximated by $f \approx 1+5 k_{B} T / 2 m c^{2}$, and the thermal correction is small. As the temperature $T$ increases, $k_{B} T \gg$ $m c^{2}$ and $f \approx 4 k_{B} T / m c^{2}$, and therefore $Q$ rapidly decreases as $T^{-5}$. In a nonmagnetized plasma, $Q=0$, and the nonlinearity of the equation vanishes in this formulation. It occurs because we are using the covariant Maxwell equation [Eq. (3)]. In the case of a nonmagnetized plasma, this wave equation is linear for $\vec{A}$ because $\gamma \vec{v}$ is proportional to $\vec{A}$ [see Eq. (10)].

\section{MODULATIONAL INSTABILITY}

Based on the nonlinear Schrödinger equation (19), we can study the stability conditions for the electromagnetic wave under a low-frequency modulation. The modulational instability can be excited when the group dispersion $P(\omega)$ and the nonlinear frequency shift $Q(\omega)$ [Eqs. (15) and (17), respectively] have the same sign [30]. In our case this condition is fulfilled.

We now consider a modulated wave packet which satisfies Eq. (19). The modulation may be expressed in terms of a slow space-time variation close to the average finite value $a_{0}$ as $z-V t \rightarrow \pm \infty$, where $V$ is the effective group velocity of the wave packet [29]. This is the reason for keeping the term proportional to $\left|a_{0}\right|^{2}$ in Eq. (19).

Notice that the above coefficients $P(\omega)$ and $Q(\omega)$ could be determined from the nonlinear dispersion relation in the weakly magnetized limit [see Eq. (18)], through the relations $P=(1 / 2) \partial^{2} \omega / \partial k^{2}$ and $Q=-\partial \omega / \partial a_{0}^{2}[1,29]$.

To study the stability of the modulation, we decompose $a$ as $a=\sqrt{\rho(z, t)} \exp [i \sigma(z, t)][1]$. Replacing this expression in

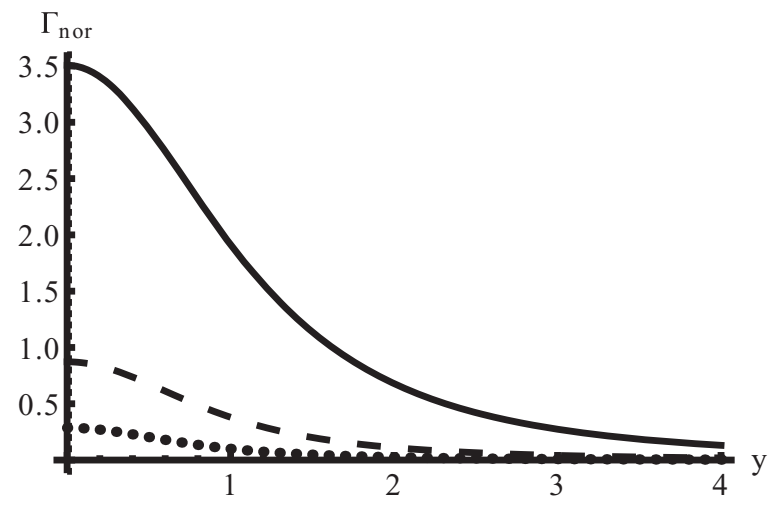

FIG. 1. Normalized maximum growth rate given by Eq. (24), in terms of $y=c k / \omega_{p}$. We use $\alpha=0.01$. Full line: cold case $(T=0)$; dashed line: $T=0.09 \mathrm{keV}$; dotted line: $T=0.18 \mathrm{MeV}$.

(19), we obtain two equations for $\rho$ and $\sigma$ :

$$
\begin{gathered}
\frac{\partial \rho}{\partial t}+2 P \frac{\partial}{\partial z}\left(\rho \frac{\partial \sigma}{\partial z}\right)=0 \\
\frac{\partial \sigma}{\partial t}+P\left[\frac{1}{4 \rho^{2}}\left(\frac{\partial \rho}{\partial z}\right)^{2}-\frac{1}{2 \rho} \frac{\partial^{2} \rho}{\partial z^{2}}+\left(\frac{\partial \sigma}{\partial z}\right)^{2}\right]=Q\left(\rho-\rho_{0}\right),
\end{gathered}
$$

where

$$
\rho_{0}=\left|a_{0}\right|^{2}
$$

To solve these equations we linearize with respect to the uniform solution,

$$
\begin{aligned}
& \rho=\rho_{0}+\rho_{1} e^{i k_{L} z-i \omega_{L} t}, \\
& \sigma=\sigma_{1} e^{i k_{L} z-i \omega_{L} t},
\end{aligned}
$$

where $\rho_{1}$ and $\sigma_{1}$ are first-order quantities, so that $\rho_{1} \ll \rho_{0}$ and $\sigma_{1} \ll \sigma_{0}$. In this way we obtain the dispersion relation for the low-frequency modulation

$$
\omega_{L}^{2}=P^{2} k_{L}^{4}-2 P Q \rho_{0} k_{L}^{2} .
$$

It is clear from Eq. (23) that the modulational instability has a threshold given by $\rho_{0}>P k_{L}^{2} / 2 Q$, and therefore, the maximum growth rate is $\Gamma=Q \rho_{0}$. The maximum growth rate and the instability depend on the temperature and on the background magnetic field. As the temperature $T$ increases so that $f \approx 4 k_{B} T / m c^{2}$, the maximum growth rate decreases as $T^{-5}$. Thus, for a very high-temperature plasma, the maximum growth rate and the nonlinearities rapidly vanish. To show this behavior, we plot in Fig. 1 a normalized maximum growth rate

$$
\Gamma_{\text {nor }}=\frac{\Gamma}{3 \lambda \omega_{p} \rho_{0}}=\frac{\alpha}{f^{5} x^{3}},
$$

where for a given normalized wave number $y=c k / \omega_{p}$ and temperature (as given by the value of $f$ ), $x$ can be calculated from the dispersion relation. In our case we will use Eq. (18).

In Fig. 1 we plot $\Gamma_{\text {nor }}$ as a function of normalized wavelength, for three different temperatures: a cold plasma (full line), $T=0.09 \mathrm{keV}$ (dashed line), and $T=0.18 \mathrm{MeV}$ (dotted line) in the $f^{2} \gg \lambda\left|a_{0}\right|^{2}$ limit. We can see that the growth rate vanishes as the temperature increases. 

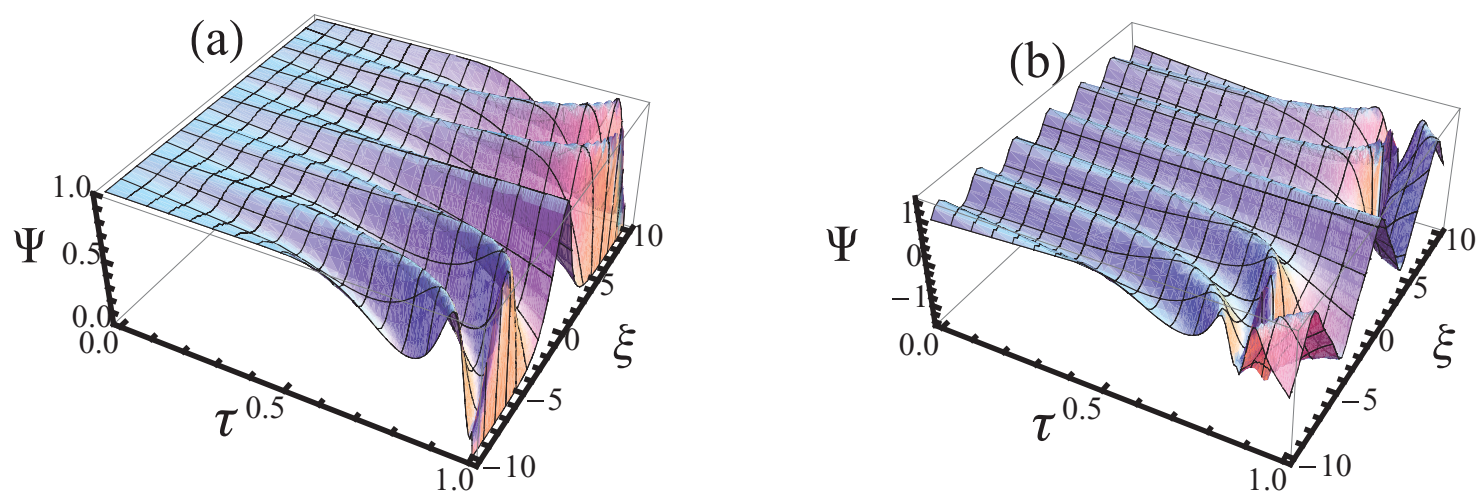

FIG. 2. (Color online) Solutions of Eq. (26) as a function of $-10 \leqslant \xi \leqslant 10$ and $0 \leqslant \tau \leqslant 1$. (a) Solutions for $\Psi(0)=1$ and $\Psi^{\prime}(0)=0$. The constant solution is for $\tau=0$, whereas a solitary wave solution exists for $\tau=1$. For values $0<\tau<1$ we find periodic wave trains. (b) Solutions for $\Psi(0)=1$ and $\Psi^{\prime}(0)=0.5$. For these values there are only periodic wave trains whose amplitude becomes bigger as the value of $\tau$ increases.

When the modulation grows, the instability evolves into a nonlinear stationary state balancing the dispersion with the nonlinearity. To study these nonlinear states, we focus on Eq. (19) for $a(z, t)$. We define the normalized variable

$$
\frac{a(z, t)}{a_{0}}=\Psi(\xi) e^{i \eta},
$$

in terms of the new variable $\Psi$, which depends on $\xi=$ $\left(Q a_{0}^{2} / 2 P\right)^{1 / 2} \chi$, where $\chi=z-V t$ and

$$
\eta=\frac{V}{2 P} z-\left(\frac{V^{2}}{4 P}+\frac{Q a_{0}^{2}}{2} \tau^{2}\right) t,
$$

with $\tau$ a parameter which can be determined later. With (25), Eq. (19) becomes

$$
\frac{d^{2} \Psi}{d \xi^{2}}=\left(2-\tau^{2}\right) \Psi-2 \Psi^{3} .
$$

The solutions of (26) depend on the values of $\Psi(0)$ and $\Psi^{\prime}(0)$ and can be written in terms of Jacobi elliptic functions where $\tau$ is the elliptic modulus [31]. In Fig. 2 we plot numerical solutions of Eq. (26), where we observe wave trains and localized solitons depending on the initial conditions and on the value of $\tau$.

By varying the initial conditions, the solution can go from periodic wave trains to localized solitons for the different values of $\tau$. In particular, when $\tau=1$ and $\Psi^{\prime}(0)=0$, we obtain a localized solitary wave solution $\Psi=\operatorname{sech}(\xi)$ [31], so that the solution of Eq. (25) for $a$ is

$$
a(z, t)=a_{0} \operatorname{sech}\left(\sqrt{\frac{Q a_{0}^{2}}{2 P}} \chi\right) e^{i \eta},
$$

and therefore

$$
\begin{aligned}
\rho & =a(z, t) a^{*}(z, t)=\rho_{0} \operatorname{sech}^{2}\left[\left(\frac{Q \rho_{0}}{2 P}\right)^{1 / 2} \chi\right] \\
& =\rho_{0} \operatorname{sech}^{2}\left[\left(\frac{3 \lambda \rho_{0}}{f^{5}}\right)^{1 / 2} \frac{\Omega_{c}}{x c} \chi\right] .
\end{aligned}
$$

The solution (28) is an envelope soliton because the envelope of the wave packet has the form of a solitary wave.
The soliton depends on the temperature (through $f$ ), the magnetic field (through $\Omega_{c}$ ), and the normalized frequency $x$ [given by Eq. (18)]. As in the case of the maximum growth rate, the nonlinear corrections vanish at large temperatures [see Eq. (17)]. In effect, the nonlinear solution (28) has a larger width as $T$ increases, becoming $\rho=\rho_{0}$ for $T \rightarrow \infty$, and thus there is no solitary wave in this limit. Likewise, if there is no background magnetic field $\left(\Omega_{c} \rightarrow 0\right)$, there is no soliton solution.

In Fig. 3 we plot the soliton solution (28) $\rho=$ $\rho_{0} \operatorname{sech}^{2}\left(\sqrt{\alpha / f^{5}} \xi_{n} / x\right)$ in terms of $\xi_{n}=\sqrt{3 \lambda \rho_{0}} \omega_{p} \chi / c$ and $k_{B} T$ [Fig. 3(a)], and in terms of $\xi_{n}$ and $y$ [Fig. 3(b)]. The normalized
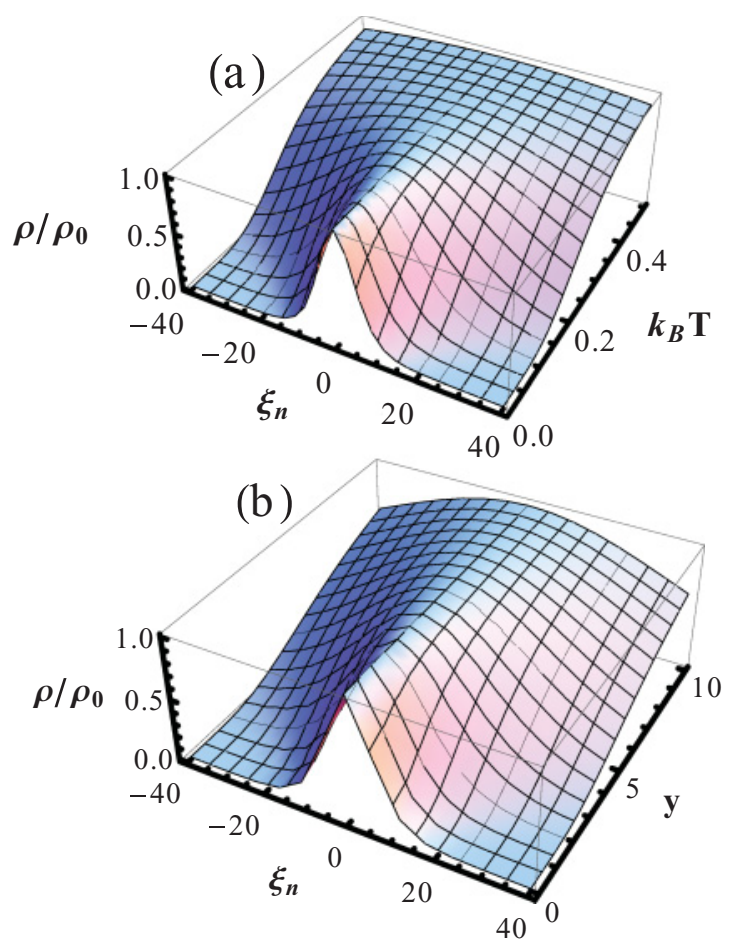

FIG. 3. (Color online) (a) The soliton solution $\rho$ in terms of $-40 \leqslant \xi_{n} \leqslant 40$ and $0 \leqslant k_{B} T \leqslant 0.5 \mathrm{MeV}$ for $y=1$, with $k_{B} T$ in $\mathrm{MeV}$. (b) Its behavior with respect to $-40 \leqslant \xi_{n} \leqslant 40$ and $0 \leqslant y \leqslant 10$ for temperature $k_{B} T=0.05 \mathrm{MeV}$. We have taken $\alpha=0.1$. 
frequency $x$ is calculated from Eq. (18), taking $\alpha=0.1$. We have taken $y=1$ for Fig. 3(a) and $k_{B} T=0.05 \mathrm{MeV}$ for Fig. 3(b).

Figure 3(a) shows how the soliton solution gets wider in $\xi_{n}$ as we increase the temperature, reducing the effect of the nonlinear corrections in Eq. (17). Eventually the localized solution becomes effectively a light mode with uniform amplitude for very large temperatures. Similarly, the solitary wave also becomes effectively a uniform solution for large wave numbers.

\section{SUMMARY}

We have derived a nonlinear Schrödinger equation for the spatiotemporal evolution of the complex amplitude of a circularly polarized electromagnetic wave in a weakly magnetized pair plasma with relativistic temperatures. We showed that the nonlinearity vanishes as the plasma gets unmagnetized or as the temperature $T \rightarrow \infty$. This is explicitly seen in the function $Q$ in Eq. (17) that reflects the nonlinearity of the Schrödinger equation, and in the maximum growth rate (24). Both functions decrease as $T^{-5}$ when the temperature increases. This effect can also be seen in Fig. 3 .
Using the complex modulation representation for the vector potential $\vec{A}$, we found the modulational instability and the maximum growth rate in terms of the background magnetic field and the temperature. We also showed that a soliton solution is allowed for the modulation. Here the temperature plays an important role in the structure of the envelope soliton because as the temperature increases the envelope soliton gets wider converging to a uniform solution for very large temperatures.

Our results may contribute to improve the understanding of physical processes in pair plasmas such as the formation and evolution of pulsar microstructures [1,32-36].

\section{ACKNOWLEDGMENTS}

F.A.A. acknowledges the hospitality of INPE and Universidad de Concepción and thanks to CONICyT for support through a BecasChile Postdoctoral Fellowship. This project has been financially supported by FONDECyT under contracts 1110135 (J.A.V.) and No. 1080658 (V.M.). A.C.L.C. and E.L.R. acknowledge support from the Brazilian agencies CNPq and FAPESP. A.C.L.C. acknowledges support through a Marie Curie International Incoming Fellowship and the hospitality of Paris Observatory.
[1] A. C.-L. Chian and C. F. Kennel, Astrophys. Space Sci. 97, 9 (1983).

[2] V. S. Beskin, A. V. Gurevich, and Y. N. Istamin, Physics of the Pulsar Magnetosphere (Cambridge University Press, Cambridge, 1993).

[3] G. Björnsson, M. A. Abramowicz, X. Chen, and J.-P. Lasota, Astrophys. J. 467, 99 (1996).

[4] H. Lesch and G. T. Bisk, Phys. Plasmas 5, 2773 (1998).

[5] S. J. Hardy and M. H. Thoma, Phys. Rev. D 63, 025014 (2000).

[6] R. Sunyaev et al., Astrophys. J. 383, L49 (1991).

[7] C. S. Reynolds, A. C. Fabian, A. Celottid, and M. J. Rees, Mon. Not. R. Astron. Soc. 283, 873 (1996).

[8] R. M. Bandyopadhyay, J. Silk, J. E. Taylor, and T. J. Maccarone, Mon. Not. R. Astron. Soc. 392, 1115 (2009).

[9] S. C. Wilks, H. Chen, E. Liang, P. Patel, D. Price, B. Remington, R. Shepherd, M. Tabak, and W. L. Kruer, Astrophys. Space Sci. 298, 347 (2005).

[10] H. Chen et al., High Energy Density Phys. 7, 225 (2011).

[11] E. N. Nerush, I. Y. Kostynkov, A. M. Fedotov, N. B. Narozhny, N. V. Elkina, and H. Ruhl, Phys. Rev. Lett. 106, 035001 (2011).

[12] W. Lünow, Plasma Phys. 10, 973 (1968).

[13] P. C. Clemmow, J. Plasma Phys. 12, 297 (1974).

[14] C. F. Kennel and R. Pellat, J. Plasma Phys. 15, 335 (1976).

[15] A. C.-L. Chian and R. B. Miranda, Lett. Nuovo Cim. 26, 249 (1979).

[16] A. C.-L. Chian, Phys. Fluids 24, 369 (1981).

[17] A. C.-L. Chian, Lett. Nuovo Cim. 29, 393 (1980).

[18] F. A. Asenjo, V. Muñoz, J. A. Valdivia, and T. Hada, Phys. Plasmas 16, 122108 (2009).

[19] M. Lontano, S. Bulanov, and J. Koga, Phys. Plasmas 8, 5113 (2001).
[20] D. Farina and S. V. Bulanov, Phys. Rev. Lett. 86, 5289 (2001).

[21] D. Farina and S. V. Bulanov, Phys. Rev. E 64, 066401 (2001).

[22] Y. Wang, Z. Zhou, H. Wang, H. Sun, H. Zhang, and R. Qin, Plasma Sci. Technol. 8, 151 (2006).

[23] N. C. Lee, Phys. Plasmas 18, 062310 (2011).

[24] D. Farina and S. V. Bulanov, Plasma Phys. Rep. 27, 641 (2001).

[25] M. Y. Yu, P. K. Shukla, and L. Stenflo, Astrophys. J. 309, L63 (1986).

[26] F. B. Rizatto, R. S. Schneider, and D. Dillenburg, Phys. Lett. A 133, 59 (1988).

[27] F. T. Gratton, G. Gnavi, R. M. O. Galvão, and L. Gomberoff, Phys. Rev. E 55, 3381 (1997).

[28] S. M. Mahajan, Phys. Rev. Lett. 90, 035001 (2003).

[29] K. Nishikawa and C. S. Liu, in Advances in Plasma Physics, edited by A. Simon and W. B. Thompson (Wiley, New York, 1976), vol. 6, chap. 2, pp. 3-81.

[30] A. Hasegawa, Plasma Instabilities and Nonlinear Effects (Springer-Verlag, Berlin, 1975).

[31] A. Ebaid and S. M. Khaled, J. Comput. Applied Math. 235, 1984 (2011).

[32] A. B. Mikhaiilovskii, O. G. Onishchenko, and A. I. Smolyakov, Soviet Astron. Lett. 11, 78 (1985) [Pis'ma Astron. Zh. 11, 190 (1985)].

[33] G. Machabeli, D. Khechinashvili, G. Melikidze, and D. Shapakidze, Mon. Not. R. Astron. Soc. 327, 984 (2001).

[34] M. V. Popov, N. Bartel, W. H. Cannon, A. Y. Novikov, V. I. Kondratiev, and V. I. Altunin, Astron. Astrophys. 396, 171 (2002).

[35] S. A. Petrova, Astron. Astrophys. 417, L29 (2004).

[36] T. H. Hankins and J. A. Eilek, Astrophys. J. 670, 693 (2007). 